\title{
Conservação refrigerada de carambolas em embalagens plásticas
}

\author{
Rosemberg G. da Cruz ${ }^{1}$, Eros C. de Almeida ${ }^{2}$, Elizete Amorim ${ }^{3}$ \& Soraia V. Borges ${ }^{4}$ \\ ${ }_{1}^{1}$ Aluno do curso de Engenharia Química - UFRRJ. Bolsista FAPERJ (Foto) \\ 2 Aluno do curso de Engenharia de Alimentos- Bolsista PIBIC-CNPq-UFRRJ \\ ${ }^{3}$ Técnica em microbiologia - DTA/UFRRJ. Fone: (21) 2682-1865 \\ ${ }^{4}$ DTA/IT/UFRRJ, km 47 da Antiga Rodovia Rio-SP, CEP 23855-970, Seropédica, RJ. E-mail: svborges@ufrrj.br
}

Protocolo $037-03 / 04 / 2000$

\begin{abstract}
Resumo: Pesquisou-se o efeito de duas embalagens (polietileno de baixa densidade - PEBD e polipropileno - Longa Vida Pak ou LVP) em relação ao controle (sem embalagem) sobre a conservação de carambolas em atmosfera refrigerada $\left(10^{\circ} \mathrm{C}\right)$. Utilizou-se o planejamento fatorial inteiramente casualizado $3 \times 4 \times 2$ ( 3 embalagens $\times 4$ períodos de armazenamento $\times 2$ repetições). As análises para acompanhamento do armazenamento foram: $\mathrm{pH}$, ${ }^{\circ}$ Brix (sólidos solúveis), acidez titulável, perda de peso, coliformes totais e fecais, bolores e leveduras. O nível de contaminação microbiológica (bolores e leveduras) foi aceitável até 21 e 28 dias para carambolas não embaladas e embaladas, respectivamente. A embalagem LVP apresentou os menores índices de contaminação. Em relação às alterações físico-químicas, não houve diferenças significativas entre as embalagens com relação a perda de peso, $\mathrm{pH}$ e acidez titulável e o teor de sólidos solúveis foi maior para os frutos armazenados em embalagens LVP. Os resultados globais indicam que ambas as embalagens aumentam a vida útil deste produto e que, do ponto de vista microbiológico, a embalagem LVP foi melhor que a embalagem PEBD para a preservação sob condições refrigeradas.
\end{abstract}

Palavras-chave: vida de prateleira, frutos, embalagem, Averrhoa carambola L.

\section{Refrigerated preservation of carambolas in plastic packagings}

\begin{abstract}
The aim of the study was to investigate the effect of two packaging materials (low density polyethylene- LPDE and polypropylene-LVP) on Averrhoa carambola L shelf-life under refrigeration. A factorial design $3 \times 4 \times 2$ (3 packaging $\times 4$ days of storage $\times 2$ repetitions) was used. The fruits were analysed for $\mathrm{pH}$, total soluble solids, total titrable acidity, weight loss, total and fecal coliforms, moulds and yeasts. The microbiological contamination levels (moulds and yeast) was acceptable up to 21 and 28 storage days for unpacked and packed fruits, respectively. The fruits inside LVP packaging showed lower level of contamination. The weight loss, $\mathrm{pH}$ and titrable acidity alterations were not significantly different between the two packagings. The total soluble solids were higher for fruits inside the LVP packaging. These global results indicate that both packagings increased the shelf-life of the product and that from a microbiological standpoint, the LVP packaging was better than the LPDE packaging for preservation under refrigerated conditions.
\end{abstract}

Key words: shelf life, fruits, packaging, Averrhoa carambola L.

\section{INTRODUÇÃO}

O consumo de frutas frescas é essencial na dieta humana, pois são alimentos ricos em vitaminas, sais minerais e fibras dietárias. A caramboleira, segundo Saúco (1994) é considerada fruteira de grande potencial mercadológico devido, dentre outros fatores, ao rápido desenvolvimento, alta produtividade, sabor e aparência peculiares. No Brasil, esta planta se adapta às nossas condições climáticas, e seu principal consumidor "in natura" é o Estado de São Paulo (Araújo \& Minami, 2001). Pode ser utilizada, também, para a fabricação de doces e geléias (Baldini et al., 1982; Jordão \& Bonas, 1995).

A carambola (Averrhoa carambola L.) atinge a maturidade fisiológica quando ligada à arvore e, após a colheita, continua seu amadurecimento para se transformar em fruto comestível e, então, passa à fase de senescência (Baldini et al., 1982). Estas fases são acompanhadas de muitas reações químicas que culminam também em transformações físicas, dentre as quais 
se destacam, para este fruto em particular, a excessiva perda de água e o ressecamento, ocasionados pela transpiração e armazenamento inadequado (Campbell \& Koch, 1989). Trata-se, também, de um alimento muito perecível, pois possui cerca de $94 \%$ de umidade (Balbach \& Boarim, 1992) o que o torna susceptível ao ataque microbiano. Estas reações deteriorativas podem ser minimizadas com o abaixamento da temperatura e com o uso de embalagens de baixa permeabilidade à água (Neves Filho, 1991). O uso de ceras (Vines \& Grierson, 1966; Sanchez, 1990) e de embalagem plástica individual (Brown et al., 1985) foi testado com sucesso para minimizar a perda de água em carambolas. Outras transformações pós-colheita, estudadas por Wan \& Lam (1984), Campbell \& Koch (1989) e Campbell et al. (1987 e 1989) mostraram que a concentração de açúcares aumenta durante a maturação mas permanece constante durante a estocagem; o comportamento dos ácidos é variado e dependente da temperatura, visto que declinam inicialmente e podem permanecer constantes ou declinar continuamente durante o armazenamento.

Devido à pouca expressão comercial desta fruta no Brasil, são raros, ou mesmo inexistentes, os trabalhos sobre pós-colheita ou conservação de carambolas nacionais; faz-se necessário, portanto, um estudo dessa natureza para que o cultivo e o processamento dessa fruta exótica e de fácil adaptação em solo brasileiro, sejam intensificados. Neste sentido, o presente trabalho tem, como objetivo, comparar a eficiência de duas embalagens: de polietileno de baixa densidade e Longa Vida Pak-propileno, em relação ao controle (sem embalagem) na conservação de carambolas frescas, por 1 mês, em ambiente refrigerado.

\section{MATERIAL E MÉTODOS}

Matérias primas: carambolas obtidas de pomares caseiros e de coloração verde, foram sanitizadas com solução de $200 \mathrm{ppm}$ de cloro, por $15 \mathrm{~min}$, secadas a temperatura ambiente e colocadas em bandejas plásticas (polietileno de alta densidade), envoltas ou não (controle) com embalagens a serem testadas, seladas, pesadas e colocadas em geladeiras, mantidas a $10 \pm 1{ }^{\circ} \mathrm{C}$, sem controle de umidade relativa.

Embalagens: polietileno de baixa densidade (PEBD) (espessura: $0,05 \mathrm{~mm}$ ) e Longa Vida Pak - polipropileno (LVP) (espessura: $0,02 \mathrm{~mm}$ ) da Wanor Indústria e Comércio Import \& Export. Ltda.

Planejamento experimental: utilizou-se planejamento fatorial completo, inteiramente casualizado: 3 tratamentos (controle, PEBD e LVP) x 4 (tempos de armazenamento) x 2 (repetições). Foram colocados 3 frutos por bandeja, cujos resultados foram analisados através do teste de Tukey (5\% de probabilidade) segundo Gomes (1990).

Análises físico-químicas: perda de peso, sólidos solúveis, acidez titulável (ácido cítrico) e pH, de acordo com metodologias descritas pela AOAC (1980).

Análises microbiológicas: coliformes fecais e totais, bolores e leveduras, conforme Speck (1984).

\section{RESULTADOS E DISCUSSÃO}

O tratamento fitossanitário foi satisfatório, pois no final do experimento o nível de coliformes fecais e totais $\left(<0,3 \mathrm{NPM} \mathrm{g}^{-1}\right)$ foi menor que o padrão máximo de segurança $\left(2 \times 10^{2}\right)$ segundo a Portaria n 451/97, do Ministério da Saúde (Brasil, 1998). Quanto a bolores e leveduras, verificou-se que a amostra-controle apresentou, após o $21^{\circ}$ dia, alto índice de contaminação ( $>3 \mathrm{x}$ $10^{5} \mathrm{UFC} \mathrm{g}^{-1}$ ) enquanto as embaladas atingiram este patamar aos 28 dias, observando-se menor contaminação para carambolas embaladas em LVP $\left(<3 \times 10^{4} \mathrm{UFC}^{-1}\right)$ em relação às embaladas em PEBD $\left(>3 \times 10^{5} \mathrm{UFC} \mathrm{g}^{-1}\right)$.

A Tabela 1 (A, B, C e D) mostra a análise de variância para as respostas analisadas nesse estudo. Observa-se, ainda, que apenas para o teor de sólidos solúveis ou ${ }^{\circ}$ Brix e perda de peso, as variáveis dias e embalagens foram significativas. A interação embalagens versus dias foi significativa para a perda de peso. Esses resultados globais sugerem que as diferenças significativas e diárias se devem ao uso exclusivo das embalagens mas, entre elas, as diferenças são mínimas ou inexistentes, conforme resumem os resultados apresentados na Tabela 2 e, portanto, ambas são adequadas para o armazenamento dessas frutas.

Tabela 1. Análise de variância

\begin{tabular}{lrrrr}
\hline Fonte de Variação & SQ & GL & QM & F \\
\hline A. Perda de peso (\%) em carambolas sob refrigeração & \\
Embalagem & 12302,1716 & 2 & 6151,3578 & $570,491^{*}$ \\
Dias & 2301,0890 & 3 & 767,0298 & $71,136^{*}$ \\
Embalagem x Dias & 3825,5656 & 6 & 637,5943 & $59,132^{*}$ \\
Resíduo & 129,3907 & 12 & 10,7826 & \\
\hline Total & 18558,2170 & 23 & \\
\hline B. Sólidos solúveis $\left(\mathrm{g} 100 \mathrm{~g}^{-1}\right)$ em carambolas sob refrigeração \\
Embalagem & 6,6307 & 1 & 6,6307 & $11,354^{*}$ \\
Dias & 12,4869 & 3 & 4,16292 & $7,130^{*}$ \\
Embalagem x Dias & 4,9819 & 3 & 1,6606 & 2,845 \\
Resíduo & 4,6700 & 8 & 0,5837 & \\
\hline Total & 28,7695 & 15 & \\
\hline
\end{tabular}

C. Acidez titulável (\% em ácido cítrico) em carambolas sob refrigeração

\begin{tabular}{|c|c|c|c|c|}
\hline Embalagem & 0,0050 & 1 & 0,0052 & 1,543 \\
\hline Dias & 0,0096 & 3 & 0,0032 & 0,931 \\
\hline Embalagem x Dias & 0,0020 & 3 & 0,0007 & 1,970 \\
\hline Resíduo & 0,0272 & 8 & 0,0030 & \\
\hline Total & 0,0438 & 15 & & \\
\hline \multicolumn{5}{|c|}{ D. pH em carambolas sob refrigeração } \\
\hline Embalagem & 1,5000 & 1 & 1,5000 & 3,622 \\
\hline Dias & 2,1103 & 3 & 0,7034 & 1,698 \\
\hline Embalagem x Dias & 1,9795 & 3 & 0,6598 & 1,593 \\
\hline Resíduo & 3,3141 & 8 & 0,4143 & \\
\hline Total & 8,9039 & 15 & & \\
\hline
\end{tabular}

* Significativo a nível de $5 \%$ de probabilidade

SQ: soma dos quadrados; QM: quadrado médio; GL: grau de liberdade; F: teste de Fischer

A Figura 1 mostra as variações na perda de peso sofridas pelos frutos armazenados. Observa-se nitidamente que o uso dessas embalagens reduz consideravelmente a perda de peso (menor que 10\%), estando de acordo com pesquisas similares na literatura (Scalon et al., 1996; Vieites et al., 1998). Em relação à carambola, Campbell et al. (1989) estudando o seu armazenamento, quando embaladas em caixa de papelão a $10{ }^{\circ} \mathrm{C}$, observaram perdas de $2 \%$ em 14 dias de estocagem, ligeiramente superiores às encontradas neste trabalho com filmes plásticos (máximo de 1,6\%); entretanto, não foram 
Tabela 2. Teste de Tukey para propriedades físico-químicas da carambola

\begin{tabular}{|c|c|c|c|c|}
\hline Embalagem & $\begin{array}{c}\text { Perda de Peso } \\
(\%)\end{array}$ & $\begin{array}{l}\text { Sólidos Solúveis } \\
\qquad\left(\mathrm{g} 100 \mathrm{~g}^{-1}\right)\end{array}$ & $\mathrm{pH}$ & $\begin{array}{c}\text { Acidez Titulável } \\
\text { (g } 100 \mathrm{~g}^{-1} \text { gácido cítrico) }\end{array}$ \\
\hline PEBD & $1,8986 \mathrm{a}$ & $7,1750 \mathrm{a}$ & $4,2875 \mathrm{a}$ & $0,2275 \mathrm{a}$ \\
\hline LVP & $0,0009 \mathrm{a}$ & $8,4625 \mathrm{~b}$ & $4,9000 \mathrm{a}$ & $0,2637 \mathrm{a}$ \\
\hline
\end{tabular}

Obs.: Letras com sinais diferentes diferem significativamente a nível de 0,05 da probabilidade

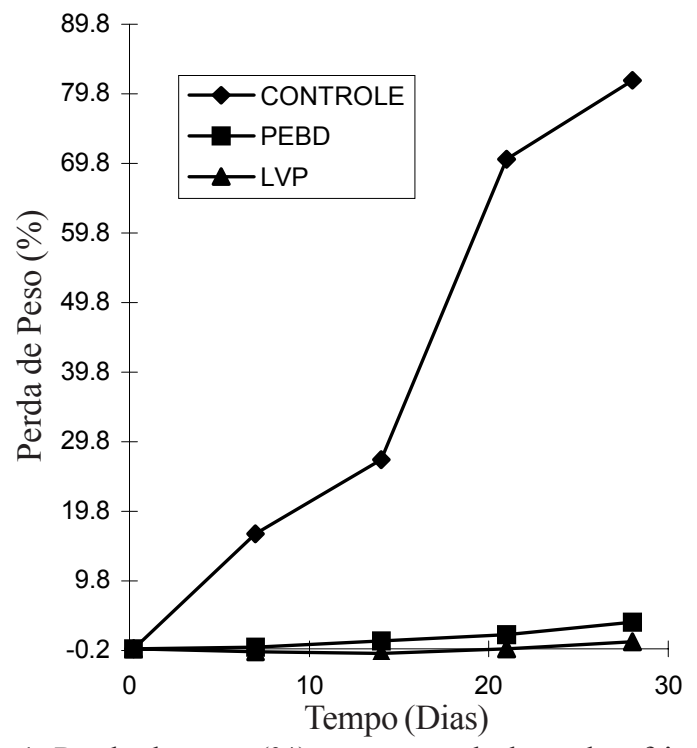

Figura 1. Perda de peso (\%) para carambolas sob refrigeração em função do tempo de armazenamento

observadas diferenças significativas entre as embalagens, conforme a Tabela 2.

A Figura 2 indica a variação de sólidos solúveis em função do tempo de armazenamento. Inicialmente, os frutos

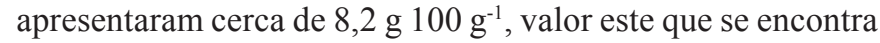
na faixa obtida por Andrews (1989) o qual estudou muitas

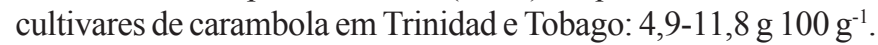
Em relação aos resultados com frutas nacionais, foi ligeiramente superior aos encontrados por Araújo \& Minami (2001):

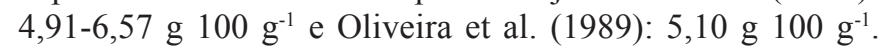
Até o sétimo dia de armazenamento observou-se aumento nos sólidos solúveis totais, mais acentuadamente para carambolas não embaladas, em decorrência das reações químicas de

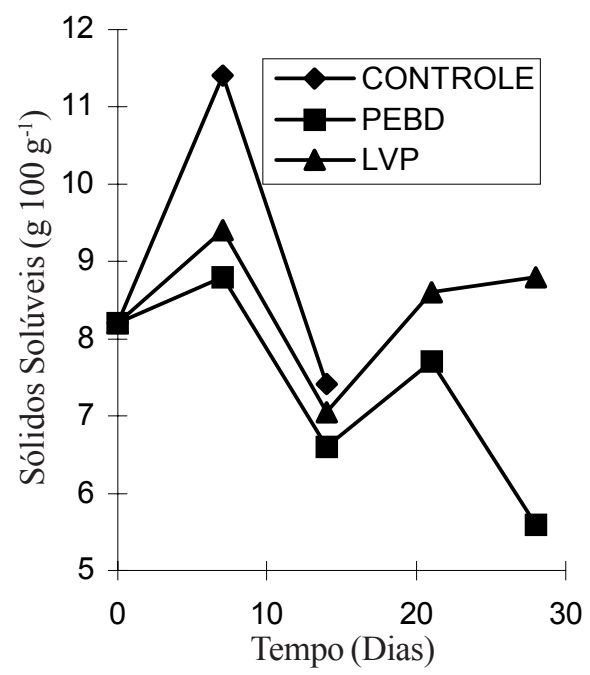

Figura 2. Sólidos solúveis para carambolas sob refrigeração em função do tempo de armazenamento amadurecimento e concomitante perda de peso. Após este período, uma queda pouco significativa é observada corroborando, desta forma, com resultados obtidos por Campbell \& Koch (1989) e Wan \& Lam (1984) os quais verificaram que os níveis de sacarose, glicose e frutose para carambolas permaneceram constantes durante a estocagem refrigerada a 5 , 10 e $15{ }^{\circ} \mathrm{C}$. Após o $21^{\circ}$ dia de armazenamento, as análises não puderam ser feitas para o controle devido à quase total desidratação do produto. A Tabela 2 mostra que houve diferenças significativas entre as embalagens para este constituinte, sendo registrada maior queda para frutos contidos na embalagem PEBD.

As Figuras 3 e 4 mostram a ligeira redução da acidez titulável e elevação do pH com o tempo de conservação. Tais relações inversas são devidas à conversão dos ácidos orgânicos não

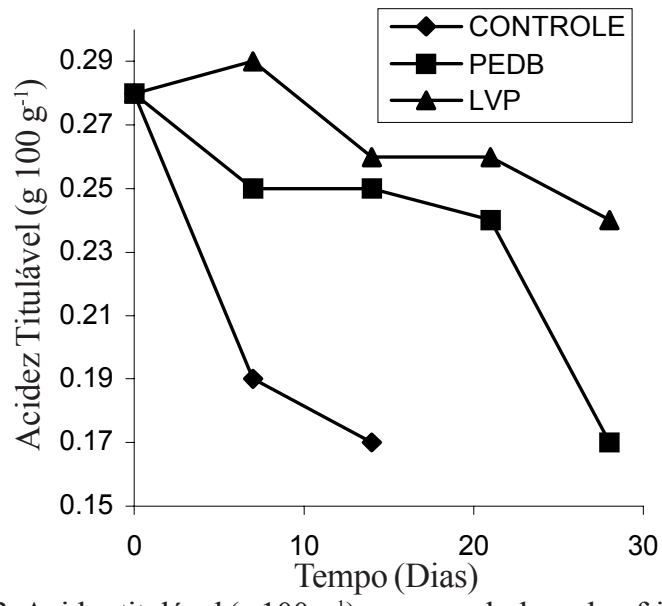

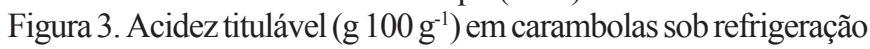
em função do tempo de armazenamento

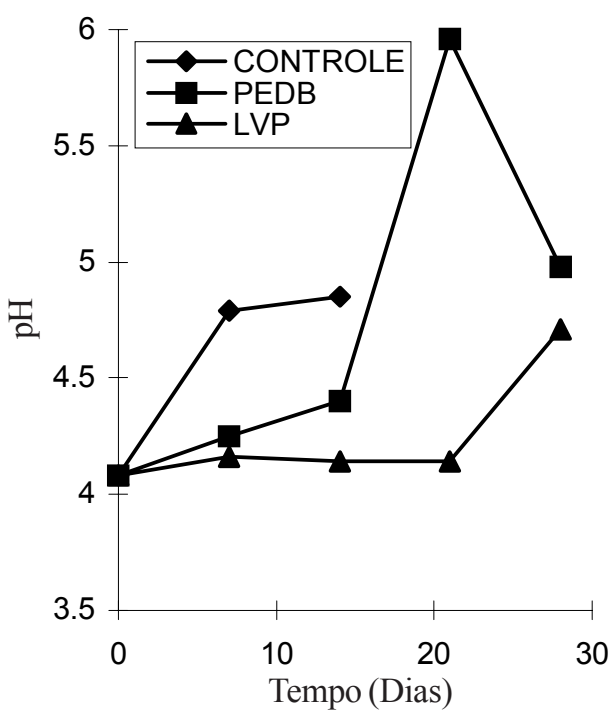

Figura 4. pH em carambolas sob refrigeração em função do tempo de armazenamento 
voláteis em $\mathrm{CO}_{2}$, açúcares e outros produtos, resultantes da atividade metabólica durante o armazenamento. Entretanto, estas variações diárias de acordo com a Tabela 1 (C e D), não foram significativas. Campbell \& Koch (1989) estudando as transformações glicídicas e acídicas de carambolas sob refrigeração a $10{ }^{\circ} \mathrm{C}$, também encontraram resultados similares. A acidez (\% em ácido cítrico) encontrada para este fruto na literatura brasileira, foi de 0,37\% (Oliveira et al., 1989), ligeiramente superior ao nosso resultado $(0,28 \%)$. O pH inicial $(4,2)$ se encontra próximo aos resultados obtidos por Tidbury \& Wagner (1988): 4,2 e 4,9, respectivamente, citados por Araújo \& Minami (2001). A análise estatística apresentada na Tabela 2 mostrou que não houve variação significativa para o pH e acidez com relação à variável embalagem.

\section{CONCLUSÕES}

1. Ambas as embalagens são adequadas ao armazenamento refrigerado de carambolas, sendo este tempo inferior a 28 dias.

2. Os resultados obtidos pelas análises físico-químicas microbiológicas sugerem a embalagem LVP, à base de polipropileno, como a mais adequada para conservação das carambolas, pois apresentou menor contaminação microbiológica e queda nos sólidos solúveis.

\section{LITERATURA CITADA}

Andrews, L. Propects for carambola production in Trinidad and Tobago. Proceedings of Interamerican Society of the Tropical Horticulture, Campeche, v.33, p.44-46, 1989.

AOAC Official methods of analysis. Washington: Association of Official Analytical Chemists, Washington D.C., 1975. p.1094

Araújo, P.S.R.; Minami, K. Seleção de caramboleiras pelas características biométricas e físico-químicas dos frutos. Scientia Agricola, v.58, n.1, p.1-17, 2001.

Balbach, A.; Boarim, D.S.F. As frutas da medicina natural. $2 \cdot$ ed. Itaquaquecetuba: Vida Plena, 1992.316p.

Baldini, V.L.S.; Draeta, I.S.; Nomura, E.H. Avaliação bioquímica de carambola (Averrhoa carambola, L.). Coletânea do ITAL, Campinas, v.12, p.283-291, 1982.

Brasil. Ministério da Saúde. Portaria n²51, de 19/09/1997. Regulamento técnico e princípios gerais para estabelecimento de critérios e padrões microbiológicos para alimentos. Anexo I. Diário Oficial da República Federal do Brasil, Brasília, 1998.

Brown, B.I.; Wong, L.S.; Watson, B.J. Use of plastic film packaging and low temperature storage for postharvest handling of rambutam, carambola and sapodilla. Proceedings Postharvest Horticultural. Workshop, Melbourne, p.272-286, 1985.
Campbell, C.A.; Huber, D.J.; Koch, K.E. Postharvest response of carambolas to storage at low temperatures. Proceedings of the Flórida State Horticultural Society, Tallahassee, v.100, p.272-275, 1987.

Campbell, C.A .; Huber, D.J. ; Koch, K.E. Postharvest changes in sugars, acids, and color of Carambola fruit at various temperatures. HortScience, Alexandria, v.24, n.3, p.472-475, 1989.

Campbell, C.A.; Koch, K.E. Sugar/acid composition and development of sweet and tart carambola fruit. Journal of the American Society Horticultural Science, Mount Vernon, v.114, n.3, p.455-457, 1989.

Gomes, F.P. Curso de estatística experimental. 13. ed., São Paulo: Nobel, 1990. 467p.

Jordão, P.R.; Bonnas, D.S. Aproveitamento industrial da carambola. Alimentos/Qualidade \& Produtividade, São Paulo, n. 7, p.28-29, 1995.

Neves Filho, L.C. Resfriamento, congelamento e estocagem dos alimentos. São Paulo: IBF/ABRAVA/SINDRATAR, 1991. $176 \mathrm{p}$.

Oliveira, M.N.; Maia, G.A.; Guedes, Z.B.L.; Guimarães, A . C.L.; Figueiredo, R.W. de. Características químicas e físicoquímicas da carambola (Averrhoa carambola L). Ciência Agronômica, Fortaleza, v. 20, p.129-133, 1989.

Sanchez, D. Keep it under an edible coat. Agriculture Research, v.38, p.4-5, 1990.

Saúco, V.G. Possibilities of no-citrus tropical fruit in the Mediterranean. Acta Horticulturae, Hague, n.365, p.25-41, 1994.

Scalon, S.P.Q.; Bittencourt, A.L.; Chitarra, A.B.; Chitarra, M.I.F. Avaliação da qualidade e da vida útil de morangos (Fragaria ananassa Duch.) submetidos à aplicação pós-colheita de $\mathrm{CaCl}_{2}$ e armazenados sob atmosfera modificada à temperatura ambiente. Ciência e Tecnologia de Alimentos, v.16, n.1, p.8387, 1996.

Speck, M.L. Compendium of methods for the microbiological examination of foods. 2.ed. Washington, USA: APHA, 1984.

Vieites, R.L.; Neves, LT.C.; Silva, A.P. Utilização da embalagem de polietileno e diferentes tipos de ceras em condições ambiente e sob refrigeração na conservação do tomate. In: Congresso Brasileiro de Ciência e Tecnologia de Alimentos, 16, 1998, Rio de Janeiro. Resumos... Rio de Janeiro: Sociedade Brasileira de Ciência e Tecnologia de Alimentos, 1998. CD-Rom

Vines, H.M.; Grierson, W. Handling and physiological studies with the carambola. Proceedings of the Florida State Horticultural Society, Tallahassee, v.79, p.350-355, 1966.

Wan, C.K.; Lam, P.F. Biochemical changes, use of polyethylene bags, and chilling injury of carambola (Averrhoa carambola L). Pertanika, Selagon Dorar Ehsan, n.7, p.39-46, 1984. 